\title{
Eliciting Constant and Prominent Waves n34-p44 of Vestibular-evoked Myogenic Potentials
}

\author{
TSUNG-WEI HUANG ${ }^{1}$, YI-HO YOUNG ${ }^{2}$ and PO-WEN CHENG ${ }^{1}$ \\ From the Departments of Otolaryngology, ${ }^{1}$ Far Eastern Memorial Hospital and ${ }^{2}$ National Taiwan University Hospital, Taipei, Taiwan
}

\begin{abstract}
Huang T-W, Young Y-H, Cheng P-W. Eliciting constant and prominent waves n34-p44 of vestibular-evoked myogenic potentials. Acta Otolaryngol 2004; 124: 1-6.

Objective - The serial peaks of vestibular-evoked myogenic potentials (VEMPs) have been labeled p13, n23, n34 and p44 according to their latency. Waves p13-n23 have been shown to be of saccular origin, whereas the origin of waves n34-p44 is still unknown. In order to improve the clinical applicability of waves $n 34-p 44$, we examine the use of different patterns of acoustic stimuli to evoke constant and prominent VEMPs, especially waves n34-p44.

Material and Methods - In this prospective study 27 healthy volunteers (54 ears) underwent VEMP tests. Three kinds of click intensity (85, 95 and $105 \mathrm{~dB} \mathrm{nHL})$ were presented in a random order to evoke 85-VEMP, 95-VEMP and 105-VEMP, respectively. The response rate, latency of each peak, peak-to-peak interval and amplitude of waves p13-n23 and n34-p44 were measured and analyzed.

Results - The response rates of waves p13-n23 in 85-VEMP, 95-VEMP and 105-VEMP were 26\% (14/54), 89\% (48/54) and $98 \%(53 / 54)$, respectively. Significant differences in the response rate existed between 85-VEMP and both 95-VEMP and $105-$ $\operatorname{VEMP}(p<0.01)$, whereas there was a non-significant difference between 95-VEMP and 105-VEMP $(p>0.05)$. In contrast, the response rates for eliciting waves n34-p44 were 19\% (10/54), 63\% (34/54) and 89\% (48/54), using 85, 95 and $105 \mathrm{~dB}$ acoustic stimuli, respectively. A significantly higher response rate for waves n34-p44 occurred when the intensity of the stimuli increased $(p<0.01)$. Although neither latencies nor interval exhibited a significant difference between 95-VEMP and 105-VEMP, the amplitude of 105-VEMP was significantly greater than that of 95-VEMP for both waves p13-n23 and n34$\mathrm{p} 44$.

Conclusion - An acoustic stimulus intensity of $105 \mathrm{~dB}$ nHL is required to reliably elicit waves $\mathrm{n} 34-\mathrm{p} 44$ in subjects with normal hearing. Key words: click, intensity, vestibular-evoked myogenic potential, wave n34-p44, wave p13-n23.
\end{abstract}

\section{INTRODUCTION}

Colebatch et al. (1) successfully applied loud sounds to evoke vestibular-evoked myogenic potentials (VEMPs) in the tonically contracted ipsilateral sternocleidomastoid (SCM) muscle, and labeled the serial peaks p13, $\mathrm{n} 23, \mathrm{n} 34$ and $\mathrm{p} 44$, based on their latencies. In contrast to the biphasic waves $\mathrm{p} 13-\mathrm{n} 23$, which are supposed to originate from the sacculo-collic reflex $(2-5)$, the origin of waves $\mathrm{n} 34-\mathrm{p} 44$ remains undetermined, although a cochlear origin has been proposed because waves $\mathrm{n} 34-\mathrm{p} 44$ could be obtained in ears after selective vestibular nerve section (1). In the past decade, VEMPs have been widely studied in several clinical diseases, such as Ménière's disease (6), cerebellopontine angle tumor (7), multiple sclerosis (8), superior canal dehiscence syndrome (9), etc. However, researchers have focused almost solely on investigating waves $\mathrm{p} 13-\mathrm{n} 23$, possibly due to the higher response rate in normal controls. In contrast, waves $\mathrm{n} 34-\mathrm{p} 44$ could be elicited in only $55-60 \%$ of healthy subjects, interrupting the investigation of their clinical significance $(1,10)$. The aim of this study was therefore to elicit steady and prominent waves $\mathrm{n} 34-\mathrm{p} 44$ with a higher response rate in normal subjects in order to improve their clinical applicability.

\section{MATERIAL AND METHODS}

Twenty-seven healthy volunteers ( 15 males, 12 females; mean age 32 years; range 21-40 years) underwent VEMP tests. All subjects denied any previous ear diseases. Surface electromyographic (EMG) activity was recorded (Medelec Synergy, Old Woking, UK) in a supine subject, with an active electrode placed on the upper half of the SCM muscle and a reference electrode placed on the lateral end of the upper sternum. During the recording, the subject was instructed to hold his/her head slightly raised in order to activate the SCM muscles. EMG activities were monitored on a display so as to maintain them at a constant level $(50-200 \mu \mathrm{V})$ in individual test ears. EMG signals were amplified and band-filtered between 20 and $2000 \mathrm{~Hz}$. Three kinds of click intensity (85, 95 and $105 \mathrm{~dB} \mathrm{nHL})$ were given through a headphone to elicit 85-VEMP, 95-VEMP and 105VEMP, respectively. The stimulation rate was $5 \mathrm{~Hz}$ and the analysis time for each stimulus was $50 \mathrm{~ms}$. In total, 128 consecutive trials to stimuli were averaged for each run. Two reproducible runs were averaged as the final response.

The initial positive/negative polarity of the waveform with peaks termed $\mathrm{p} 13$ and $\mathrm{n} 23$ based on their 
latencies was used to determine the presence or absence of waves p13-n23. The subsequent negative/ positive polarity of the waveform was defined as peaks $\mathrm{n} 34$ and p44 according to their latencies. The relative amplitude indicated the amplitude of 95-VEMP divided by that of 105-VEMP in either waves p13$\mathrm{n} 23$ or $\mathrm{n} 34-\mathrm{p} 44$ for the same test ear. The response rate, latency of each peak, peak-to-peak interval and amplitude of waves p13-n23 and n34-p44 were measured and analyzed. Comparative analysis of these results was conducted using McNemar's test, a twotailed paired $t$-test and the Wilcoxon signed-rank test (11). $p<0.05$ was considered significant. This study was approved by the institutional review board and each subject gave their informed consent to participate.

\section{RESULTS}

\section{Waves p13-n23}

All 27 normal volunteers (54 ears) completed VEMP tests using various click stimuli. The response rates for eliciting waves p13-n23 using 85, 95 and $105 \mathrm{~dB}$ acoustic stimuli were $26 \%$ (14/54), 89\% (48/54) and $98 \%$ (53/54), respectively. Significant differences in the response rate existed between 85-VEMP and both 95VEMP and 105-VEMP $(p<0.01$; McNemar's test), whereas there was a non-significant difference between 95-VEMP and 105-VEMP ( $p>0.05$; McNemar's test; Fig. 1A). Excluding six ears with absent waves p13n23 in 95-VEMP, a total of 48 ears were compared. The mean latencies of waves p13 and $\mathrm{n} 23$ and the peak-to-peak interval of 95-VEMP were $11.91 \pm 0.94$, $19.09 \pm 1.38$ and $7.18 \pm 1.54 \mathrm{~ms}$, respectively, whereas those of 105 -VEMP were $11.90 \pm 0.93,19.12 \pm 1.41$ and $7.21 \pm 1.21 \mathrm{~ms}$, respectively. There was a nonsignificant difference between 95-VEMP and 105-VEMP for all of these parameters $(p>0.05$; two-tailed paired $t$-test; Table I). The minimum, maximum and median amplitudes of waves p13-n23 in 95-VEMP were 44.20, 240.65 and $93.90 \mu \mathrm{V}$, respectively, and the corresponding values for $105-$ VEMP were 43.35, 297.9 and $122.50 \mu \mathrm{V}$. The minimum, maximum and median relative amplitudes were $44 \%, 130 \%$ and $84 \%$, respectively (Table II). Since the tonic EMG activities were maintained at a constant level only in individual ear recordings, Wilcoxon signed-ranks in amplitudes p13-n23 of 105-VEMP -95-VEMP on the same testing ears, rather than the absolute amplitudes, were used for analysis. Hence, the amplitude p13-n23 of 105-VEMP was significantly larger than that of 95-VEMP $(p<0.01$; Wilcoxon signed-rank test).

\section{Waves $n 34-p 44$}

The response rates for eliciting waves $\mathrm{n} 34-\mathrm{p} 44$ were $19 \%$ (10/54), 63\% (34/54) and 89\% (48/54) using 85, 95 and $105 \mathrm{~dB}$ acoustic stimuli, respectively. A significantly higher response rate for waves $\mathrm{n} 34-\mathrm{p} 44$ occurred when the intensity of the stimuli increased $(p<$ 0.01; McNemar's test; Fig. 1B). After excluding 20 ears with absent waves n34-p44 in either 95-VEMP or 105-VEMP, the remaining 34 ears were compared and analyzed. The mean latencies of waves n 34 and p44 and the peak-to-peak interval of 95-VEMP were $29.98 \pm 2.28,37.64 \pm 2.87$ and $7.66 \pm 1.79 \mathrm{~ms}$, respectively, whereas those of 105-VEMP were $30.16 \pm 2.43$, $37.56 \pm 2.95$ and $7.40 \pm 1.64 \mathrm{~ms}$, respectively; these

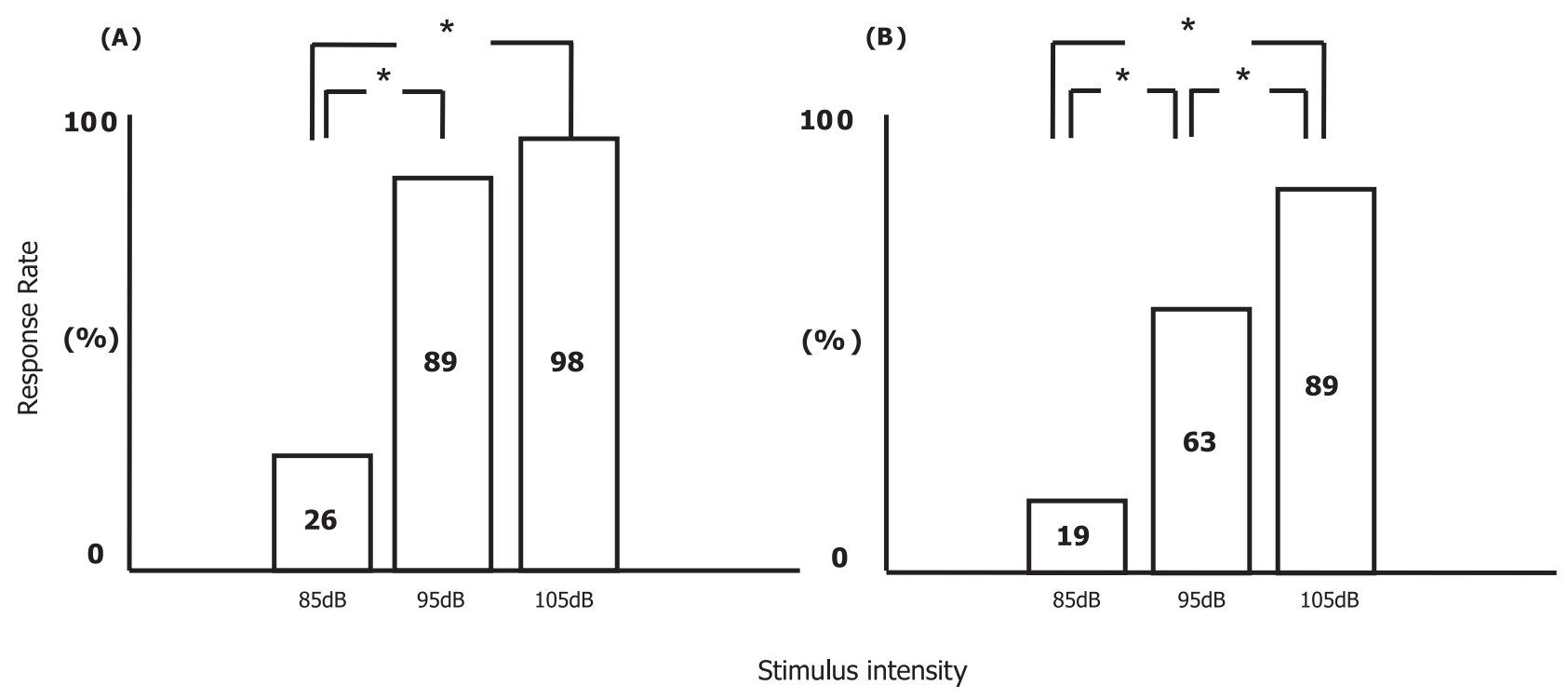

Fig. 1. Comparison of response rates of VEMPs using varied acoustic stimuli: (A) waves p13-n23; (B) waves n34-p44. Asterisk indicates $p<0.01$. 
Table I. Latencies and interval of waves p13-n23 in 95-VEMP and 105-VEMP. Results are expressed as mean \pm SD

\begin{tabular}{lllll}
\hline & No. of ears & Latency p13 $(\mathrm{ms})$ & Latency n23 $(\mathrm{ms})$ & $\begin{array}{l}\text { Interval } \\
\text { p13-n23 (ms) }\end{array}$ \\
\hline 95-VEMP & 48 & $11.91 \pm 0.94$ & $19.09 \pm 1.38$ & $7.18 \pm 1.54$ \\
105-VEMP & 48 & $11.90 \pm 0.93$ & $19.12 \pm 1.41$ & $7.21 \pm 1.21$ \\
$p$ & & NS & NS & NS \\
\hline
\end{tabular}

Table II. Amplitude and relative amplitude of waves p13-n23 elicited using various click stimuli

\begin{tabular}{llllll}
\hline & No. of ears & Minimum & Maximum & Median & IQR \\
\hline Amplitude p13-n23 $(\mu \mathrm{V})$ & & & & & \\
95-VEMP & 48 & 44.20 & 240.65 & 93.90 & 51.46 \\
105-VEMP & 48 & 43.35 & 297.90 & 122.50 & 71.46 \\
Relative amplitude (\%) & 48 & 44 & 130 & 84 & 27 \\
\hline
\end{tabular}

$\mathrm{IQR}=$ inter-quartile range.

differences were not significant $(p>0.05$; two-tailed paired $t$-test; Table III). The minimum, maximum and median amplitudes of waves n34-p44 in 95-VEMP were $21.30,178.45$ and $62.35 \mu \mathrm{V}$, respectively, and the corresponding values for 105-VEMP were 26.10, 186.09 and $62.95 \mu \mathrm{V}$. The minimum, maximum and median relative amplitudes were $41 \%, 159 \%$ and $95 \%$, respectively (Table IV). The amplitude n34-p44 of 105-VEMP was significantly greater than that of 95$\operatorname{VEMP}(p=0.03$; Wilcoxon signed-rank test).

\section{Waves $p 13-n 23$ versus waves $n 34-p 44$}

For comparing waves p13-n23 and n34-p44, a total of 34 ears that presented both waves were statistically analyzed. The peak-to-peak intervals of waves p13$\mathrm{n} 23$ and $\mathrm{n} 34-\mathrm{p} 44$ were $7.18 \pm 1.52$ and $7.66 \pm 1.79 \mathrm{~ms}$, respectively in 95-VEMP, compared to $7.24 \pm 1.50$ and $7.40 \pm 1.64 \mathrm{~ms}$, respectively in 105-VEMP; the differences between waves p13-n23 and n34-p44 in either 95-VEMP or 105-VEMP were not significant $(p>$ 0.05 ; two-tailed paired $t$-test). The amplitude of waves p13-n23 was significantly greater than that of waves n34-p44 in either 95-VEMP or 105-VEMP $(p<0.01$; Wilcoxon signed-rank test; Table V).

\section{DISCUSSION}

The definite origin of waves $n 34-p 44$ is still unknown. On the one hand, as waves $n 34-\mathrm{p} 44$ could be obtained in ears after selective vestibular nerve section, it was proposed that they probably arose from cochlear afferents (1). On the other hand, waves n34-p44 could also be obtained in deaf ears (10), implying that they were probably not of cochlear origin only. Taking these results together suggests that waves n34-p44 may have both a cochlear and vestibular origin. One possible pathway to explain this dual origin, in addition to the cochlear afferent, is the vestibulocochlear projection, which has been proven to arise from saccular afferents to the cochlear nucleus in guinea pigs $(12,13)$. Another question is whether waves $\mathrm{n} 34-\mathrm{p} 44$ are related to the startle reflex. The acoustic startle reflex is a relatively simple motor response characterized by rapid habituation and a latency of $\approx 50 \mathrm{~ms}$, in contrast to the higher rates of repetition and shorter latency (Table III) of waves n34-p44 (14). Consequently, the startle reflex would not seem to be involved in waves n34-p44. Further electrophysiological or pathological study is necessary

Table III. Latencies and interval of waves n34-p44 in 95-VEMP and 105-VEMP. Results are expressed as mean \pm $S D$

\begin{tabular}{lllll}
\hline & & & & Interval \\
& No. of ears & Latency $\mathrm{n} 34(\mathrm{~ms})$ & Latency $\mathrm{p} 44(\mathrm{~ms})$ & $7.66 \pm 1.79$ \\
\hline 95-VEMP & 34 & $29.98 \pm 2.28$ & $37.64 \pm 2.87$ & $7.40 \pm 1.64$ \\
105-VEMP & 34 & $30.16 \pm 2.43$ & $37.56 \pm 2.95$ & NS \\
$p$ & & NS & NS & \\
\hline
\end{tabular}


Table IV. Amplitude and relative amplitude of waves n34-p44 elicited using various click stimuli

\begin{tabular}{llllll}
\hline & No. of ears & Minimum & Maximum & Median & IQR \\
\hline Amplitude n34-p44 $(\mu \mathrm{V})$ & & & & & \\
95-VEMP & 34 & 21.30 & 178.45 & 62.35 & 39.60 \\
105-VEMP & 34 & 26.10 & 186.09 & 62.95 & 42.72 \\
Relative amplitude $(\%)$ & 34 & 41 & 159 & 95 & 28 \\
\hline
\end{tabular}

$\mathrm{IQR}=$ inter-quartile range.

to elucidate the mechanism of waves n34-p44, so that they can be applied to study labyrinthine or retrolabyrinthine disorders in the future.

In order to evoke constant and prominent VEMPs, many researchers have attempted to establish the optimum stimulus for VEMPs. In our laboratory, the ideal stimulus pattern for evoking waves p13-n23 by short tone bursts (STBs) is as follows: frequency 500 $\mathrm{Hz}$; stimulation repetition rate $5 \mathrm{~Hz}$; rise/fall time 1 ms; plateau time $2 \mathrm{~ms}(15-17)$. However, the ideal stimulus intensity for eliciting waves $\mathrm{p} 13-\mathrm{n} 23$ and n34-p44 remains undetermined. Because clicks seem better than STBs for evoking marked VEMPs with a higher response rate in healthy subjects (18), we used clicks instead of STBs as acoustic stimuli in this study.

When the stimulus intensity was incremented, the response rate of waves p13-n23 increased from $26 \%$ in 85 -VEMP to $89 \%$ in 95 -VEMP, and this difference was significant $(p<0.01)$. However, no statistical difference in response rate was noted between 95-VEMP $(89 \%)$ and 105-VEMP (98\%), indicating that $95 \mathrm{~dB}$ is the minimum acoustic stimulus level to yield constant waves $\mathrm{p} 13-\mathrm{n} 23$. In contrast to waves $\mathrm{p} 13-\mathrm{n} 23$, the response rate of waves $\mathrm{n} 34-\mathrm{p} 44$ was increased significantly from $63 \%$ in 95 -VEMP to $89 \%$ in 105 VEMP, meaning that $105 \mathrm{~dB}$ acoustic stimuli are required for eliciting constant waves n34-p44 (Fig. 2).

In both biphasic waves $\mathrm{p} 13-\mathrm{n} 23$ and $\mathrm{n} 34-\mathrm{p} 44$, neither latencies nor interval exhibited a significant difference between 95-VEMP and 105-VEMP (Tables I and III), which differed from the result of a previous report (19) that a lower stimulus intensity evoked responses with a statistically shorter latency of waves p13-n23. Although a decreasing wave latency following an increasing stimulus intensity was demonstrated in auditory brainstem responses (ABRs) (20), it was not observed in VEMPs in our study. One possible explanation for this observation is the difference between ABRs (neurogenic potentials) and VEMPs (myogenic potentials). Furthermore, both waves p13$\mathrm{n} 23$ and n34-p44 were recorded from the same muscle, which may explain why no significant difference in peak-to-peak interval was exhibited between them in either 95-VEMP or 105-VEMP.

In terms of amplitude, we kept the tonic EMG activity at a constant level $(50-200 \mu \mathrm{V})$ by means of EMG monitoring during VEMP recording and the testing sequence of acoustic intensity was performed in a random order in order to exclude the linear effect of tonic EMG activity on VEMP amplitude (21). Our data disclosed that both amplitudes p13-n23 and n34-p44 in 95-VEMP decreased significantly compared to those in 105-VEMP. However, the effect of stimulus intensity was more significant on the amplitude of waves p13-n23 than on that of waves n34$\mathrm{p} 44$, as a $16 \%$ reduction was seen in the relative amplitude of waves p13-n23, compared to only a 5\% reduction in that of waves n34-p44 (Tables II and IV).

Compared to waves p13-n23, not only the longer latency but also the smaller amplitude of waves n34p44 indicated that different neural pathways were involved. Waves p13-n23 of VEMPs represent the

Table V. Amplitude of waves p13-n23 and n34-p44 in 95-VEMP and 105-VEMP

\begin{tabular}{llllrl}
\hline Amplitude $(\mu \mathrm{V})$ & No. of ears & Minimum & Maximum & Median & IQR \\
\hline 95-VEMP & & & & & \\
Waves p13-n23 & 34 & 44.20 & 240.65 & 93.90 & 47.82 \\
Waves n34-p44 & 34 & 21.30 & 178.45 & 62.35 & 39.60 \\
105-VEMP & 34 & 43.35 & 297.70 & 122.50 & 63.69 \\
Waves p13-n23 & 34 & 26.10 & 186.09 & 62.95 & 42.72 \\
Waves n34-p44 & & & & \\
\hline
\end{tabular}

$\mathrm{IQR}=$ inter-quartile range 

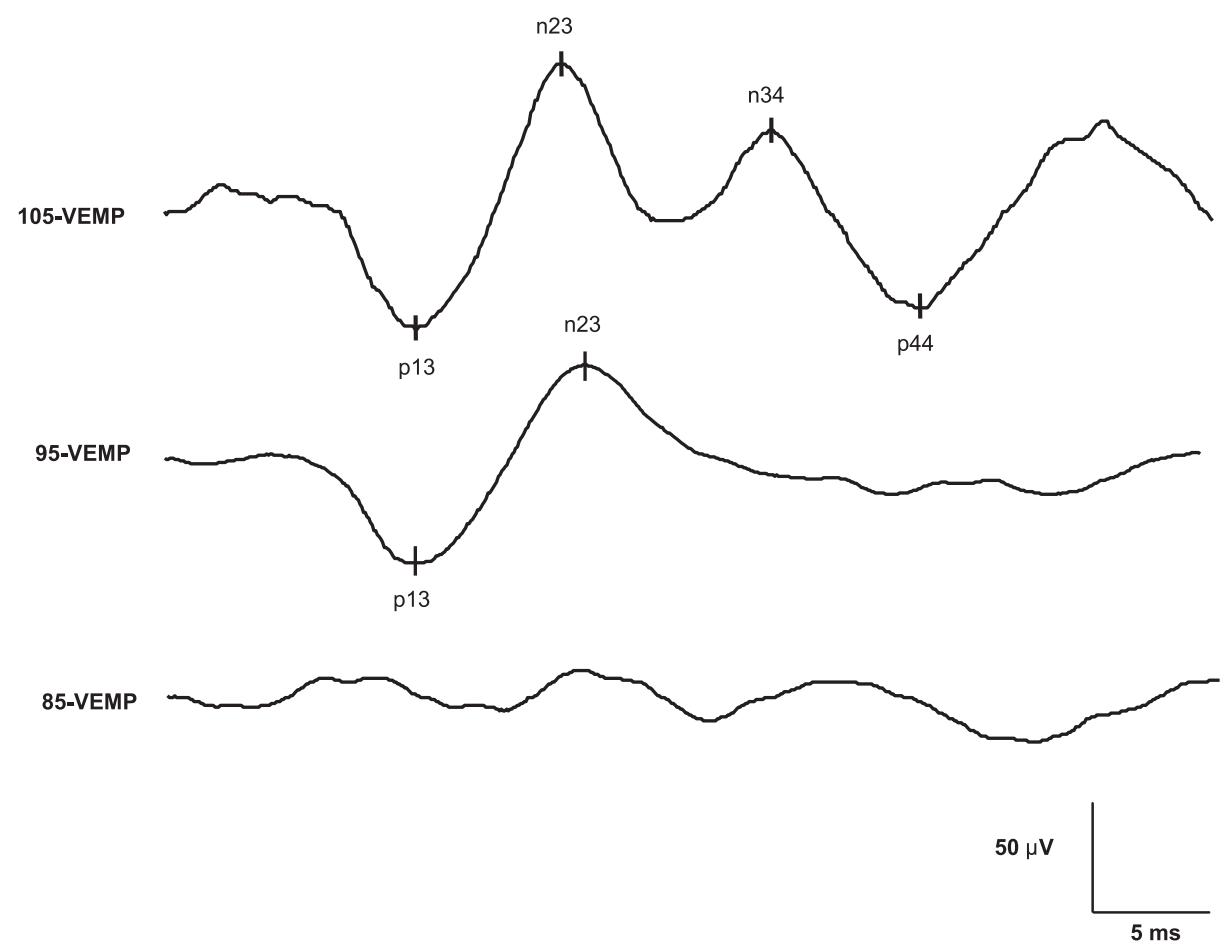

Fig. 2. VEMPs elicited in the right ear of a 30 -year-old woman. Waves p13-n23 were observed for both 95-VEMP and 105-VEMP, compared to waves n34-p44 only for 105-VEMP. No waveform was identified in 85-VEMP.

sacculo-collic reflex, which is generated via a disynaptic pathway beginning in the saccular macula and then runs via the inferior vestibular nerve, lateral vestibular nucleus and medial vestibulospinal tract, before finally terminating in the motor neurons of the SCM muscle $(1,3,5,22,23)$; i.e. vestibulocollic neurons are monosynaptically excited from the ipsilateral saccule and terminate on neck motoneurons. In contrast, the latency of waves $\mathrm{n} 34-\mathrm{p} 44$ was much longer than that of waves p13-n23 but they had similar peak-to-peak intervals, implying that the former might occur via a polysynaptic pathway, also terminating on the motor neuron of SCM muscles. As monosynaptic effects of vestibulospinal fibers, both excitatory and inhibitory, are most powerful in neck motoneurons (24), it would be anticipated that the amplitude of p13-n23 would be larger than that of n34-p44 (Table V).

In conclusion, the clinical application of waves $\mathrm{n} 34-\mathrm{p} 44$ is limited due to their lower response rate compared to that of waves $\mathrm{p} 13-\mathrm{n} 23$ in healthy subjects. Based on this study, we recommend that $105 \mathrm{~dB} \mathrm{nHL}$ acoustic stimuli are required to reliably elicit waves n34-p44 in subjects with normal hearing.

\section{REFERENCES}

1. Colebatch JG, Halmagyi GM, Skuse NF. Myogenic potentials generated by a click-evoked vestibulocollic reflex. J Neurol Neurosurg Psychiatry 1994; 57: 190-7.
2. Murofushi T, Halmagyi GM, Yavor RA, Colebatch JG. Absent vestibular evoked myogenic potentials in vestibular neurolabyrinthitis. Arch Otolaryngol Head Neck Surg 1996; 122: 845-8.

3. Uchino Y, Sato H, Sasaki M, Imagawa M, Ikegami $H$, Isu N, et al. Sacculocollic reflex arcs in cats. J Neurophysiol 1997; 77: 3003-12.

4. Todd NPM, Cody FWJ, Banks JR. A saccular origin of frequency tuning in myogenic vestibular evoked potentials? Implications for human responses to loud sounds. Hear Res 2000; 141: 180-8.

5. Kushiro K, Zakir M, Sato H, Ono S, Ogawa Y, Meng H, et al. Saccular and utricular inputs to single vestibular neurons in cats. Exp Brain Res 2000; 131: 406-15.

6. Young YH, Wu CC, Wu CH. Augmentation of vestibular evoked myogenic potentials: an indication for distended saccular hydrops. Laryngoscope 2002; 112: 509-12.

7. Chen CW, Young YH, Tseng HM. Preoperative versus postoperative role of vestibular-evoked myogenic potentials in cerebellopontine angle tumor. Laryngoscope 2002; 112: 267-71.

8. Shimizu K, Murofushi T, Sakurai M, Halmagyi M. Vestibular evoked myogenic potentials in multiple sclerosis. J Neurol Neurosurg Psychiatry 2000; 69: 276-7.

9. Streubel SO, Cremer PD, Carey JP, Weg N, Minor LB. Vestibular-evoked myogenic potentials in the diagnosis of superior canal dehiscence syndrome. Acta Otolaryngol Suppl 2001; 545: 41-9.

10. Wu CC, Young YH. Vestibular evoked myogenic potentials are intact after sudden deafness. Ear Hear 2002; 23: 235-8.

11. Glantz SA. Primer of biostatistics, 4th ed. New York: McGraw-Hill; 1997. p. 32-367. 
12. Burian M, Gstoettner W, Zundritsch R. Saccular afferent fibers to the cochlear nucleus in the guinea pig. Arch Otorhinolaryngol 1989; 246: 238-41.

13. Gstoettner W, Burian M, Zundritsch R, Mayr R. The origin of the vestibulo-cochlear projection in the guinea pig. Neurosci Lett 1991; 122: 163-6.

14. Bickford RG, Jacobson JL, Cody DT. Nature of average evoked potentials to sound and other stimuli in man. Ann N Y Acad Sci 1964; 112: 204-23.

15. Murofushi T, Matsuzaki M, Wu CH. Short tone burstevoked myogenic potentials on sternocleidomastoid muscle. Are these potentials also of vestibular origin? Arch Otolaryngol Head Neck Surg 1999; 125: 660-4.

16. Cheng PW, Murofushi T. The effect of rise/fall time on vestibular-evoked myogenic potential triggered by short tone bursts. Acta Otolaryngol 2001; 121: 696-9.

17. Cheng PW, Murofushi T. The effects of plateau time on vestibular-evoked myogenic potentials triggered by tone bursts. Acta Otolaryngol 2001; 121: 935-8.

18. Cheng PW, Huang TW, Young YH. The influence of clicks versus short tone bursts on the vestibular evoked myogenic potentials. Ear Hear 2003; 24: 195-7.

19. Ochi K, Ohashi T, Nishino H. Variance of vestibularevoked myogenic potentials. Laryngoscope 2001; 111: $522-7$.

20. Jacobson JT, Novotny GM, Elliott S. Clinical considerations in the interpretation of auditory brainstem response audiometry. J Otolaryngol 1980; 9: 493-504.
21. Lim CL, Clouston P, Sheean G, Yiannikas C. The influence of voluntary EMG activity and click intensity on the vestibular click evoked myogenic potential. Muscle Nerve 1995; 18: 1210-3.

22. Murofushi T, Curthoys IS, Gilchrist DP. Response of guinea pig vestibular nucleus neurons to clicks. Exp Brain Res 1996; 111: 149-52.

23. Wilson VJ, Boyle R, Fukushima K, Rose PK, Shinoda Y, Sugiuchi Y, et al. The vestibulocollic reflex. J Vestib Res 1995; 5: 147-70.

24. Wilson VJ, Yoshida M. Comparison of effects of stimulation of Deiters' nucleus and medial longitudinal fasciculus on neck, forelimb, and hindlimb motoneurons. J Neurophysiol 1969; 32: 743-58.

Submitted October 17, 2003; accepted January 15, 2004

Address for correspondence:

Po-Wen Cheng, MD

Department of Otolaryngology

Far Eastern Memorial Hospital

21, Section 2, Nan-Ya South Road

Pan Chiao 220

Taipei

Taiwan

Tel.: +8862 29546200, ext. 4201

Fax: +886229579505

E-mail: powenjapan@yahoo.com.tw 